International Mathematical Forum, Vol. 8, 2013, no. 31, 1523 - 1530

HIKARI Ltd, www.m-hikari.com

http://dx.doi.org/10.12988/imf.2013.37142

\title{
Axioms of Countability in Generalized Topological Spaces
}

\author{
John Benedict T. Ayawan ${ }^{1}$ \\ Sciences Cluster \\ University of the Philippines Cebu \\ 6000 Cebu City, Philippines \\ jbenz321@gmail.com \\ Sergio R. Canoy, Jr. \\ Department of Mathematics \\ College of Science and Mathematics, MSU-IIT \\ 9200 Iligan City, Philippines \\ serge_canoy@yahoo.com
}

Copyright (c) 2013 John Benedict T. Ayawan and Sergio R. Canoy, Jr. This is an open access article distributed under the Creative Commons Attribution License, which permits unrestricted use, distribution, and reproduction in any medium, provided the original work is properly cited.

\begin{abstract}
The different axioms of countability are generalized in such a way that topologies are replaced by generalized topologies in the sense of [1]. In particular, the concepts such as $\mu$-first countable, $\mu$-second countable, and $\mu$-separable are defined, where $\mu$ is a generalized topology on a nonempty set $X$.
\end{abstract}

Keywords: generalized topological space, $\mu$-first countable, $\mu$-second countable, $\mu$-separable

\section{Introduction}

A generalization of the concept of topology is that of generalized topology defined in [2]. Specifically, a subset $\mu$ of the power set $\exp X$ of $X$ is a generalized

\footnotetext{
${ }^{1}$ Research supported in part by the DOST-ASTHRDP, Philippines.
} 
topology (briefly GT) on $X$ if $\emptyset \in \mu$ and every union of elements of $\mu$ belongs to $\mu$. From this definition, it follows that every topology on $X$ is a generalized topology. The elements of a generalized topology $\mu$ on $X$ are called $\mu$-open sets. The union of all the elements of $\mu$ is denoted by $M_{\mu}$. A generalized topology is said to be strong if $M_{\mu}=X$.

The purpose of this paper is to define first countability, second countability, and separability in generalized topological spaces. We consider some properties of these concepts and characterize first countability and second countability of the product of GT's.

\section{Preliminaries}

Let $(X, \mu)$ be a generalized topological space. We say that $M \subset X$ is $\mu$-open if $M \in \mu ; N \subset X$ is $\mu$-closed if $X-N \in \mu$. If $A \subseteq X$, then $i_{\mu} A$ is the union of all $\mu$-open sets contained in $A$ and $c_{\mu} A$ is the intersection of all $\mu$-closed sets containing $A$ (see [3]). It is known that $c_{\mu} A \subseteq c_{\mu} B$, whenever $A \subseteq B$ (see [5]). If $c_{\mu} A=X$, then $A$ is said to be $\mu$-dense in $X$ [4]. Let $\mathrm{B} \subseteq \exp X$ and $\emptyset \in \mathrm{B}$. Then $\mathrm{B}$ is a base for $\mu$ if $\left\{\bigcup_{B^{*} \in \mathrm{B}_{1}} B^{*}: \mathrm{B}_{1} \subseteq \mathrm{B}\right\}=\mu[6]$. We also say that $\mu$ is generated by $\mathrm{B}$. It is shown in [5] that $\mathrm{B}$ is a base for $\mu$ iff whenever $U$ is a $\mu$-open set and $x \in U$, there exists $B \in \mathrm{B}$ such that $x \in B \subseteq U$. A class $\mathrm{B}_{p}$ of $\mu$-open sets containing $p$ is called a $\mu$-local base at $p$, if for each $\mu$-open set $U$ containing $p$, there is $U_{p} \in \mathrm{B}_{p}$ with $p \in U_{p} \subseteq U$.

\section{$3 \mu$-first countable, $\mu$-second countable and $\mu$-separable spaces}

Definition 3.1 A GTS $(X, \mu)$ is called

(1) a $\mu$-first countable space if there is a countable $\mu$-local base at every $p \in M_{\mu}$.

(2) a $\mu$-second countable space if there is a countable base for the generalized topology $\mu$.

(3) a $\mu$-separable space if $X$ contains a countable dense subset.

Example 3.2 Let $X=\mathbb{R}$ and let $\mu=\mathrm{A}_{1} \cup \mathrm{A}_{2} \cup\{\emptyset, \mathbb{R}\}$ where,

$$
\begin{aligned}
& \mathrm{A}_{1}=\left\{\left(-\infty, a_{1}\right]: a_{1} \geq 2\right\} \cup\left\{\left(-\infty, a_{2}\right): a_{2} \geq 2\right\} \text { and } \\
& \mathrm{A}_{2}=\left\{\left[b_{1},+\infty\right): b_{1} \leq 1\right\} \cup\left\{\left(b_{2},+\infty\right): b_{2} \leq 1\right\}
\end{aligned}
$$

By the Completeness Axiom, it is easy to show that $(\mathbb{R}, \mu)$ is a GTS. Observe that since $(-\infty, 3] \cap[a,+\infty)=[1,3] \notin \mu,(\mathbb{R}, \mu)$ is not a topological space. 
Now, for each $x \in M_{\mu}=\mathbb{R}$, consider the following families:

$$
\begin{aligned}
& \mathrm{B}_{x}=\{(-\infty, x],(1,+\infty)\} \text { if } x \geq 2, \\
& \mathrm{~B}_{x}=\{[x,+\infty),(-\infty, 2)\} \text { if } x \leq 1 \text { and } \\
& \mathrm{B}_{x}=\{(-\infty, 2),(1,+\infty)\} \text { if } 1<x<2
\end{aligned}
$$

It can be be verified that for each case, $\mathrm{B}_{x}$ is $\mu$-local base at $x$. Thus $(\mathbb{R}, \mu)$ is a $\mu$-first countable space. Also, since $\mathbb{Q}$ is a countable $\mu$-dense subset of $\mathbb{R}$, $(\mathbb{R}, \mu)$ is a $\mu$-separable space.

Example 3.3 Let $X=\mathbb{R}$ and let $\mu=\mathrm{A}_{1} \cup \mathrm{A}_{2} \cup\{\emptyset, \mathbb{R}\}$, where

$$
\begin{aligned}
& \mathrm{A}_{1}=\{(b,+\infty): b \leq 1\} \text { and } \\
& \mathrm{A}_{2}=\{(-\infty, a): a \geq 2\} .
\end{aligned}
$$

Again, the Completeness Axiom can be used to show that $(\mathbb{R}, \mu)$ is a GTS. Moreover, the collection $\mathrm{B}=\{\emptyset\} \cup\left\{\left(q_{1},+\infty\right): q_{1} \in \mathbb{Q}\right.$ and $\left.q_{1} \leq 1\right\} \cup$ $\left\{\left(-\infty, q_{2}\right): q_{2} \in \mathbb{Q}\right.$ and $\left.\left.q_{2} \geq 2\right)\right\}$ is a countable base for the GTS $(\mathbb{R}, \mu)$. Therefore, $(\mathbb{R}, \mu)$ is a $\mu$-second countable space.

Theorem 3.4 Let B be a base for a generalized topology $\mu$ in $X$ and $p \in M_{\mu}$. Then $\mathrm{B}_{p}=\{B \in \mathrm{B}: p \in B\}$ is a $\mu$-local base at $p$.

Proof. Suppose that B is a base for a generalized topology $\mu$ in $X$. Let $U \in \mu$ and $p \in U$. Set $\mathrm{B}_{p}=\{B \in \mathrm{B}: p \in B\}$. Since $\mathrm{B}$ is a base, there exists a subclass $\mathrm{B}^{*}$ of $\mathrm{B}$ such that $U=\bigcup_{B \in \mathrm{B}^{*}} B$. Since $p \in U$, there exists $B$ such that $p \in B$. Thus, $B \in \mathrm{B}_{p}$ and $p \in B \subseteq U$. Therefore, $\mathrm{B}_{p}$ is a $\mu$-local base at $p$.

Theorem 3.5 The following statements are equivalent:

(i) $D$ is $\mu$-dense in $X$.

(ii) If $F$ is $\mu$-closed and $D \subseteq F$, then $F=X$.

(iii) If $\mathrm{B}$ is a base for $\mu$ and $B \in \mathrm{B}$, where $B \neq \emptyset$, then $B \cap D \neq \emptyset$.

Proof. $(i) \Rightarrow($ ii $)$ Note that $D \subseteq F$ implies $c_{\mu}(D) \subseteq c_{\mu}(F)$. Since $D$ is $\mu$-dense in $X, X=c_{\mu}(D) \subseteq c_{\mu}(F)=F$.

(ii) $\Rightarrow$ (iii) Suppose that $B \cap D=\emptyset$ for some nonempty $B \in \mathrm{B}$. Then $D \subseteq B^{c}$. Since $B^{c}$ is $\mu$-closed, $B^{c}=X$, by assumption. This implies that $B=\emptyset$, contrary to the assumption of $B$.

$($ iii $) \Rightarrow(i)$ Suppose that $D$ is not $\mu$-dense in $X$. This means that $c_{\mu}(D)=T=\cap\{F: F$ is $\mu$-closed with $D \subseteq F\} \neq X$. Hence, there exists $x \in X \backslash T$. This implies that there exists a $\mu$-closed set $F$ with $D \subseteq F$ 
such that $x \notin F$. Since $F^{c}$ is a $\mu$-open set and $x \in F^{c}$, there exists $B \in \mathrm{B}$ such that $x \in B \subseteq F^{c}$. Consequently, $B \cap D \subseteq F^{c} \cap D$. Since $F^{c} \cap D=\emptyset$, it follows that $B \cap D=\emptyset$. This contradicts our assumption. Therefore, $D$ is $\mu$-dense in $X$.

Theorem 3.6 Every $\mu$-second countable generalized topological space is $\mu$ first countable.

Proof. Suppose that $X$ is $\mu$-second countable. Then $X$ has a countable base B. Let $p \in M_{\mu}$. By Theorem 3.4, $\mathrm{B}_{p}=\{B \in \mathrm{B}: p \in B\}$ is a $\mu$-local base at $p$. Since $\mathrm{B}$ is countable and $\mathrm{B}_{p} \subseteq \mathrm{B}$ it follows that $\mathrm{B}_{p}$ is a countable $\mu$-local base at $p$. This shows that $X$ is a $\mu$-first countable space.

Remark 3.7 The converse of Theorem 3.6 is not true.

To see this, consider the $\mu$-first countable GTS $(\mathbb{R}, \mu)$ in Example 3.2. Let $\mathrm{S}=\{[b,+\infty): b \leq 1\} \cup\{(-\infty, a]: a \geq 2\}$ and let $\mathrm{B}$ be a base for $\mu$. Then $\mathrm{S}$ is uncountable. Note that $b \in[b,+\infty)$ and the only $\mu$-open set containing $b$ which is contained in $[b,+\infty)$ is the set itself. Hence, $[b,+\infty) \in \mathrm{B}$ for each $b \in \mathbb{R}$ with $b \leq 1$. Similarly, $(-\infty, a] \in \mathrm{B}$ for each $a \in \mathbb{R}$ with $a \geq 2$. Thus, $\mathrm{B} \supseteq \mathrm{S}$. Therefore, $\mathrm{B}$ is uncountable. This shows that $(X, \mu)$ is not $\mu$-second countable.

Theorem 3.8 Every $\mu$-second countable generalized topological space is $\mu$ separable.

Proof. Let $(X, \mu)$ be $\mu$-second countable space and let $\mathrm{B}=\left\{B_{n}: n \in \mathbb{N}\right\}$ be a countable base for $\mu$. Assume that $B_{1}=\emptyset$. For each $n \in \mathbb{N} \backslash\{1\}$ choose a point $a_{n} \in B_{n}$. The set $A=\left\{a_{n}: n \in \mathbb{N} \backslash\{1\}\right\}$ is also countable. Note that $A \cap B_{n} \neq \emptyset$ since $a_{n} \in A \cap B_{n}$ for all $n \in \mathbb{N} \backslash\{1\}$. By Theorem 3.5, $A$ is $\mu$-dense in $X$. Therefore, $(X, \mu)$ is $\mu$-separable.

$(\mathbb{R}, \mu)$ in Example 3.2 is $\mu$-separable but not $\mu$-second countable by Remark 3.7. From this, the following remark is immediate.

Remark 3.9 The converse of Theorem 3.8 is not true.

Remark $3.10 \mu$-first countability does not imply $\mu$-separability.

Consider $X=\mathbb{R}, \mu=\left\{A: A \subseteq \mathbb{Q}^{c}\right\}$. Clearly, $\mu$ is a generalized topology but not a topology for $X=\mathbb{R}$ since $\mathbb{R} \notin \mu$. Let $p \in \mathbb{Q}^{c}$. Then $\{p\} \in \mu$. Clearly, $\{\{p\}\}$ is $\mu$-local base at $p$. Hence, every $p \in \mathbb{Q}^{c}=M_{\mu}$ has a finite $\mu$-local base at $p$. Thus, $(X, \mu)$ is $\mu$-first countable. Let $D$ be a $\mu$-dense set in $(X, \mu)$. Then $D \cap A \neq \varnothing \forall A \in \mu$. Let $p \in \mathbb{Q}^{c}$. Since $\{p\} \in \mu,\{p\} \cap D \neq \varnothing$, i.e., $p \in D$. Thus, $\mathbb{Q}^{c} \subseteq D$. Since $\mathbb{Q}^{c}$ is uncountable, it follows that $D$ is uncountable. This shows that $(X, \mu)$ is not separable. 
Remark $3.11 \mu$-separability does not imply $\mu$-first countability.

To see this, let $X=\mathbb{R}$, and let $\mu$ be the generalized topology generated by the base $B=\{(r, r+2): r \in \mathbb{R})\} \cup\{\varnothing\}$. By density property of $\mathbb{Q}$, $(r, r+2) \cap \mathbb{Q} \neq \varnothing$ for each $r \in \mathbb{R}$. By Theorem 3.5, $\mathbb{Q}$ is $\mu$-dense in $\mathbb{R}$. Thus, $(\mathbb{R}, \mu)$ is $\mu$-separable. Next let $\mathrm{B}_{p}$ be a $\mu$-local base at $p \in \mathbb{R}$. Consider $(r, r+2)$, where $r<p<r+2$. Then $(r, r+2)$ is a $\mu$-open set containing $p$. Since $\mathrm{B}_{p}$ is a $\mu$-local base at $p$, there exists $B \in \mathrm{B}_{p}$ such that $p \in B \subseteq$ $(r, r+2)$. Now, since the only $\mu$-open set $B$ satisfying this is $(r, r+2)$ itself, $B=(r, r+2)$. It follows that $\{(r, r+2): r<p<r+2\} \subseteq \mathrm{B}_{p}$. Since $\{(r, r+2): r<p<r+2\}=\{(r, r+2): r \in(p-2, p)\}$ is uncountable, $\mathrm{B}_{p}$ is uncountable. Therefore, $(\mathbb{R}, \mu)$ is not $\mu$-first countable.

\section{Product of Generalized Topologies}

Let $K \neq \emptyset$ be an index set and $\left(X_{k}, \mu_{k}\right)(k \in K)$ a class of GTS's and $X=$

$\prod_{k \in K} X_{k}$ the cartesian product of the sets $X_{k}$. Let B be the collection of all sets of the form $\prod_{k \in K} M_{k}$, where $M_{k} \in \mu_{k}$ and $M_{k}=M_{\mu_{k}}$ for all but a finite number of indices $k$. We call $\mu=\mu(\mathrm{B})$ having B as a (defining) base the product of the GT's $\mu_{k}$ (see [3]). The GTS $(X, \mu)$ is called the product of the GTS's $\left(X, \mu_{k}\right)$.

The following results play important roles in the theorems that follow.

Proposition 4.1 [3] Let $\left\{\left(X_{k}, \mu_{k}\right): k \in I\right\}$ be a collection of generalized topological spaces, $(X, \mu)$ the product of these GTS's and $A=\prod_{k \in K} A_{k}$, where $A_{k} \subseteq X_{k}$. Then $c_{\mu}(A)=\prod_{k \in K} c_{\mu_{k}}\left(A_{k}\right)$

Proposition 4.2 [3] Let $\left\{\left(X_{i}, \mu_{i}\right): i \in I\right\}$ be a collection of generalized topological spaces and let $(X, \mu)$ be the product of these GTS's. Then for each fixed $j \in I$, the projection $p_{j}:(X, \mu) \rightarrow\left(X_{j}, \mu_{j}\right)$ is $\left(\mu, \mu_{j}\right)$-open.

Proposition 4.3 [3] Let $(X, \mu)$ be the product of the GTS's $\left(X_{i}, \mu_{i}\right)$, where $i \in I$. If each $\mu_{i}$ is strong, then for each fixed $j \in I$, the projection $p_{j}$ : $(X, \mu) \rightarrow\left(X_{j}, \mu_{j}\right)$ is $\left(\mu, \mu_{j}\right)$-continuous.

Theorem 4.4 Let $\left\{\left(X_{i}, \mu_{i}\right): i \in I\right\}$ be a collection of strong generalized topological spaces and let $(X, \mu)$ be the product of these GTS's. Then $(X, \mu)$ is $\mu$-first countable if and only if each $\left(X_{i}, \mu_{i}\right)$ is $\mu_{i}$-first countable and for all but countably many indices $i, \mu_{i}=\left\{\emptyset, X_{i}\right\}$. 
Proof. Suppose that $\mu_{i}$ is strong for each $i \in I$ and $(X, \mu)$ is $\mu$-first countable. Let $\left(X_{j}, \mu_{j}\right)$ be one of the component spaces and let $x_{j}$ be an arbitrary element of $X_{j}$. Then there is an $x \in X$ such that $p_{j}(x)=x_{j}$, where $p_{j}$ is the $j$ th projection from $X$ to $X_{j}$. By assumption, there is a countable $\mu$-local base at $x$, say $\left\{U_{n}: n \in \mathbb{N}\right\}$. Observe that each $p_{j}\left(U_{n}\right)$ is $\mu_{j}$-open by Proposition 4.2. Now, let $x_{j} \in V \in \mu_{j}$. Then $p_{j}^{-1}(V)$ is a $\mu$-open set containing $x$ since $p_{j}$ is $\left(\mu, \mu_{j}\right)$-continuous by Proposition 4.3. Since the $U_{n}^{\prime} s$ form a $\mu$-local base at $x$, there is an $n \in \mathbb{N}$ such that $x \in U_{n} \subseteq p_{j}^{-1}(V)$. This implies that $x_{j} \in p_{j}\left(U_{n}\right) \subseteq V$. So the collection $\left\{p_{j}\left(U_{n}\right): n \in \mathbb{N}\right\}$ is a $\mu_{j}$-local base at $x_{j}$. This shows that each $X_{j}$ is $\mu_{j}$-first countable.

Next, assume that $J=\left\{j \in I: \mu_{j} \neq\left\{\emptyset, X_{j}\right\}\right\}$ is uncountable. For each $j \in J$, choose a $\mu_{j}$-open set $U_{j} \neq X_{j}$ and $x_{j} \in U_{j}$. Then $\left\{U_{j}: j \in J\right\}$ is uncountable. For each $i \in I \backslash J$, pick $y_{i} \in X_{i}$. Let $x=\left\langle z_{i}\right\rangle_{i \in I} \in X$, where $z_{i}=x_{i}$ for $i \in J$ and $z_{i}=y_{i}$ for each $i \in I \backslash J$. Since $X$ is $\mu$-first countable and $x \in M_{\mu}=X$ ( since each $\mu_{i}$ is strong), there is a countable $\mu$-local base $\mathrm{B}_{x}=\left\{V_{n}: n \in \mathbb{N}\right\}$ at $x$. Let $\mathrm{B}$ be the defining base for $\mu$. For each $n \in \mathbb{N}$, there exists $W_{n} \in \mathrm{B}$ such that $W_{n} \subseteq V_{n}$. Hence, $p_{j}\left(W_{n}\right) \subseteq p_{j}\left(V_{n}\right)$ for all $j \in I$ and for each $n \in \mathbb{N}$. Since $p_{j}\left(W_{n}\right)=X_{j}$ for all but finitely many $j \in I$, it follows that $p_{j}\left(V_{n}\right)=X_{j}$ for all but finitely many $j \in I$ and for each $n \in \mathbb{N}$. For any particular $n \in \mathbb{N}$, let $I_{n}=\left\{i \in I: p_{i}\left(V_{n}\right) \neq X_{i}\right\}$. Then $I_{n}$ is finite for all $n \in \mathbb{N}$. Let $S=\bigcup_{n \in \mathbb{N}} I_{n}$. Then $S \subseteq I$ and is countable. Since $J$ is uncountable, there exists $k \in J \backslash S$ such that $x_{k} \in U_{k} \neq X_{k}$. Now, $p_{k}^{-1}\left(U_{k}\right)$ is a $\mu$-open set by Proposition 4.3 and $x \in p_{k}^{-1}\left(U_{k}\right)$. Since $k \notin S, p_{k}\left(V_{n}\right)=X_{k}$ for all $n \in \mathbb{N}$. This implies that there exists no $V_{n} \in \mathrm{B}_{x}$ such that $x_{k} \in p_{k}\left(V_{n}\right)=X_{k} \subseteq U_{k}$. Hence, $\mathrm{B}_{x}$ is not a $\mu$-local base at $x$, contrary to our assumption. Therefore, $\mu_{j} \neq\left\{\emptyset, X_{j}\right\}$ for countably many $j \in I$.

For the converse, suppose that each $X_{i}$ is $\mu_{i}$-first countable and that $\mu_{i}=$ $\left\{\emptyset, X_{i}\right\}$ for all but countably many indices $i \in I$. Let $C=\left\{i \in I: \mu_{i} \neq\right.$ $\left.\left\{\emptyset, X_{i}\right\}\right\}$. Then $C$ is countable and $X=\prod_{i \in C} X_{i} \times \prod_{i \in I \backslash C} X_{i}$. Let $x=\left\langle x_{i}\right\rangle_{i \in I} \in$ $M_{\mu}=X$. For each $i \in C$, let $\mathrm{B}_{x_{i}}=\left\{U_{i, n}: n \in \mathbb{N}\right\}$ be a countable $\mu_{i}$-local base at $x_{i}$. For any finite subset of $C \times \mathbb{N}$ of the form $F=\left\{\left(i_{1}, k_{1}\right),\left(i_{2}, k_{2}\right), \ldots,\left(i_{m}, k_{m}\right)\right\}$, define $V_{F}=\prod_{j=1}^{m} M_{i_{j}, k_{j}} \times \prod_{i \in I \backslash T} X_{i}$, where $T=\left\{i_{1}, i_{2}, \ldots, i_{m}\right\}$ and $M_{i_{j}, k_{j}}=U_{i_{j}, k_{j}} \in$ $\mu_{i_{j}}$ for each $i_{j} \in T$. Set $\mathrm{B}_{x}=\left\{V_{F}: F\right.$ is a finite subset of $\left.C \times \mathbb{N}\right\}$. Then $V_{F}$ is $\mu$-open and $x \in V_{F}$ for each finite subset $F$ of $C \times \mathbb{N}$. Let $U \in \mu$ with $x \in U$. Then there exists $B \in \mathrm{B}$, where $\mathrm{B}$ is the defining base for $\mu$, such that $x \in B \subseteq U$. Then $B=\prod_{j \in J} M_{j} \times \prod_{i \in I \backslash J} X_{i}$, where $J$ is a finite subset of $C$ and $M_{j}=V_{j} \in \mu_{j}$ for each $j \in J$. For each $j \in J, x_{j}=p_{j}(x) \in p_{j}(B)=V_{j}$. Since 
$\mathrm{B}_{x_{j}}$ is a $\mu_{j}$-local base at $x_{j}$, there exists $U_{j, k_{j}} \in \mathrm{B}_{x_{j}}$ such that $x_{j} \in U_{j, k_{j}} \subseteq V_{j}$. Let $V=\prod_{j \in J} U_{j, k_{j}} \times \prod_{i \in I \backslash J} X_{i}$. Then $V \in \mathrm{B}_{x}$ and $x \in V \subseteq B \subseteq U$. This implies that $\mathrm{B}_{x}$ is a countable $\mu$-local base at $x$. Therefore, $X$ is $\mu$-first countable.

Theorem 4.5 Let $\left\{\left(X_{i}, \mu_{i}\right): i \in I\right\}$ be a collection of strong generalized topological spaces and let $(X, \mu)$ be the product of these GTS's. Then $(X, \mu)$ is $\mu$-second countable if and only if each $\left(X_{i}, \mu_{i}\right)$ is $\mu_{i}$-second countable and for all but countably many indices $i, \mu_{i}=\left\{\emptyset, X_{i}\right\}$.

Proof. Suppose that $\mu_{i}$ is strong for each $i \in I$ and $(X, \mu)$ is $\mu$-second countable. Let $\left(X_{j}, \mu_{j}\right)$ be one of the component spaces. By assumption, there is a countable base for $\mu$, say $\mathrm{B}^{*}=\left\{B_{n}: n \in \mathbb{N}\right\}$. Note that each $p_{j}\left(B_{n}\right)$ is $\mu_{j}$-open by Proposition 4.2. Let $U_{j}$ be a $\mu_{j}$-open set and $x_{j} \in U_{j}$. Since $p_{j}$ is onto there is an $x \in X$ such that $p_{j}(x)=x_{j}$, where $p_{j}$ is the $j$ th projection map from $X$ to $X_{j}$. By Proposition 4.3, $p_{j}^{-1}\left(U_{j}\right)$ is a $\mu$-open set with $x \in p_{j}^{-1}\left(U_{j}\right)$. Since $\mathrm{B}^{*}$ is a base for $\mu$ there exists $n \in \mathbb{N}$ such that $x \in B_{n} \subseteq p_{j}^{-1}\left(U_{j}\right)$. This implies that $x_{j} \in p_{j}\left(B_{n}\right) \subseteq U_{j}$. So the collection $\left\{p_{j}\left(B_{n}\right): n \in \mathbb{N}\right\}$ is a countable base for $\mu_{j}$ This shows that each $X_{j}$ is $\mu_{j}$-second countable.

Next, assume that $J=\left\{j \in I: \mu_{j} \neq\left\{\emptyset, X_{j}\right\}\right\}$ is uncountable. Since $B_{n} \in \mathrm{B}^{*}$ for all $n \in \mathbb{N}, p_{j}\left(B_{n}\right)=X_{j}$ for all but finitely many $j \in I$ and for each $n \in \mathbb{N}$. For any $n \in \mathbb{N}$, let $I_{n}=\left\{i \in I: p_{i}\left(B_{n}\right) \neq X_{i}\right\}$. Then $I_{n}$ is finite for all $n \in \mathbb{N}$. Let $S=\bigcup_{n \in \mathbb{N}} I_{n}$. Then $S \subseteq I$ and is countable. Since $J$ is uncountable, there exists $k \in J \backslash S$ such that $x_{k} \in U_{k} \neq X_{k}$. Now $p_{j}^{-1}\left(U_{k}\right)$ is a $\mu$-open set by Proposition 4.3 and $x \in p_{j}^{-1}\left(U_{k}\right)$. Since $k \notin S$, $p_{k}\left(B_{n}\right)=X_{k}$ for all $n \in \mathbb{N}$. This implies that there exists no $B_{n} \in \mathrm{B}$ such that $x_{k} \in p_{k}\left(B_{n}\right)=X_{k} \subseteq U_{k}$. Hence, $\mathrm{B}^{*}$ is not a base for $\mu$, contrary to our assumption. Therefore, $\mu_{j} \neq\left\{\emptyset, X_{j}\right\}$ for countably many $j \in I$.

For the converse, suppose that each $X_{i}$ is $\mu_{i}$-second countable and that $\mu_{i}=\left\{\emptyset, X_{i}\right\}$ for all but countably many $i \in I$. Let $C=\left\{i \in I: \mu_{i} \neq\left\{\emptyset, X_{i}\right\}\right\}$. Then $C$ is countable and $X=\prod_{i \in C} X_{i} \times \prod_{i \in I \backslash C} X_{i}$. For each $i \in C$, let $\mathrm{B}_{i}=$ $\left\{U_{i, n}: n \in \mathbb{N}\right\}$ be a countable base for $\mu_{i}$. For any finite subset of $C \times \mathbb{N}$ of the form $F=\left\{\left(i_{1}, k_{1}\right),\left(i_{2}, k_{2}\right), \ldots,\left(i_{m}, k_{m}\right)\right\}$, define $V_{F}=\prod_{j=1}^{m} M_{i_{j}, k_{j}} \times \prod_{i \in I \backslash T} X_{i}$, where $T=\left\{i_{1}, i_{2}, \ldots, i_{m}\right\}$ and $M_{i_{j}, k_{j}}=U_{i_{j}, k_{j}} \in \mu_{i_{j}}$ for each $i_{j} \in T$. Set $\mathrm{B}^{*}=\left\{V_{F}: F\right.$ is a finite subset of $\left.C \times \mathbb{N}\right\}$. Let $U \in \mu$ and $x \in U$. Then there exists $B \in \mathrm{B}$, where $\mathrm{B}$ is the defining base for $\mu$, such that $x \in B \subseteq U$. Then $B=\prod_{j \in J} M_{j} \times \prod_{i \in I \backslash J} X_{i}$, where $J$ is a finite subset of $C$ and $M_{j}=\bar{V}_{j} \in \mu_{j}$ for each $j \in J$. For each $j \in J, x_{j}=p_{j}(x) \in p_{j}(B)=V_{j}$. Since $\mathrm{B}_{j}$ is base for $\mu_{j}$, 
there exists $U_{j, k_{j}} \in \mathrm{B}_{j}$ such that $x_{j} \in U_{j, k_{j}} \subseteq V_{j}$. Let $V=\prod_{j \in J} U_{j, k_{j}} \times \prod_{i \in I \backslash J} X_{i}$. Then $V \in \mathrm{B}^{*}$ and $x \in V \subseteq B \subseteq U$. This implies that $\mathrm{B}^{*}$ is a countable base for $\mu$. Therefore, $X$ is $\mu$-second countable.

Theorem 4.6 Let $\left\{\left(X_{i}, \mu_{i}\right): i \in I\right\}$ be a collection of strong generalized topological spaces and let $(X, \mu)$ be the product of these GTS's. Then $(X, \mu)$ is $\mu$-separable if and only if each $\left(X_{j}, \mu_{j}\right)$ is $\mu_{j}$-separable.

Proof. Let $D$ be a countable $\mu$-dense subset of $X$ and $B_{j}$ be a nonempty member of a base $\mathrm{B}_{j}$ for $\mu_{j}$. By Proposition 4.3 and the fact that $p_{j}$ is onto, $p_{j}^{-1}\left(B_{j}\right)$ is a non-empty $\mu$-open set. Hence $p_{j}^{-1}\left(B_{j}\right) \cap D \neq \emptyset$ by Theorem 3.5. Thus,

$$
\emptyset \neq p_{j}\left(p_{j}^{-1}\left(B_{j}\right) \cap D\right) \subseteq p_{j}\left(p_{j}^{-1}\left(B_{j}\right)\right) \cap p_{j}(D)=B_{j} \cap p_{j}(D) .
$$

This shows that $p_{j}(D)$ is $\mu_{j}$-dense in $X_{j}$. Also, since $D$ is countable $p_{j}(D)$ is countable. Therefore, $X_{j}$ is $\mu_{j}$-separable.

Suppose that each $X_{j}$ is $\mu_{j}$-separable. For each $j \in J$, let $D_{j}$ be a countable $\mu_{j}$-dense subset of $X_{j}$. Then $\prod_{j \in J} D_{j}$ is countable. By Proposition 4.1, $c_{\mu}\left(\prod_{j \in J} D_{j}\right)=\prod_{j \in J} c_{\mu}\left(D_{j}\right)=\prod_{j \in J} X_{j}=X$. Therefore, $X$ is $\mu$-separable.

\section{References}

[1] Á. Császár, Generalized Open Sets in Generalized Topologies, Acta Math Hungar.,106 (2005), 53-66.

[2] Á. Császár, Generalized Topology, Generalized Continuity, Acta Math Hungar.,96 (2002), 351-357.

[3] Á. Császár, Product of Generalized Topologies,Acta Math Hungar.,123 (2009), 127-132.

[4] E. Ekici,Generalized Hyperconnectedness, Acta Math Hungar.,133 (2011), 140-147.

[5] R. Khayyeri, R. Mohamadian, On Base for Generalized Topological Space, Int. J. Contemp. Math. Sciences,48 (2011), 2377 - 2383.

[6] G.E. Xun, G. E. Ying, $\mu$-Separations in Generalized Topological Spaces, Appl. Math. J. Chinese Univ., 25(2010), 243-252.

Received: July 11, 2013 